Research paper

\title{
The association of COVID-19 infection in pregnancy with preterm birth: A retrospective cohort study in California
}

Deborah Karasek, PhD, MPH ${ }^{\mathrm{a}, \mathrm{b}, *}$, Rebecca J. Baer, MPH ${ }^{\mathrm{b}, \mathrm{c}}$, Monica R. McLemore, PhD, MPH, RN, FAAN ${ }^{\mathrm{d}}$, April J. Bell, PhD, MPH ${ }^{\mathrm{a}, \mathrm{b}}$, Bridgette E. Blebu, PhD, MPH ${ }^{\mathrm{a}, \mathrm{b}}$, Joan A. Casey, PhD ${ }^{\mathrm{e}}$, Kimberly Coleman-Phox, MPH ${ }^{\mathrm{a}, \mathrm{b}}$, Jean M. Costello ${ }^{\mathrm{b}, \mathrm{f}}$, Jennifer N. Felder, PhD ${ }^{\mathrm{g}, \mathrm{o}}$, Elena Flowers, PhD, RN ${ }^{h}$, Jonathan D. Fuchs, MD, MPH ${ }^{\mathrm{b}, \mathrm{i}}$, Anu Manchikanti Gomez, PhD, MSc $^{j}$, Kayla Karvonen, MD ${ }^{k}$, Miriam Kuppermann, PhD, MPH ${ }^{\mathrm{a}, \mathrm{b}}$, Liang Liang, $\mathrm{PhD}^{1}$, Safyer McKenzie-Sampson, MSPH ${ }^{\mathrm{m}}$, Charles E. McCulloch, $\mathrm{PhD}^{\mathrm{m}}$, Scott P. Oltman, MS ${ }^{\mathrm{b}, \mathrm{m}}$, Matthew S Pantell, MD, MS ${ }^{\mathrm{b}, \mathrm{k}}$, Xianhua Piao, MD, PhD ${ }^{\mathrm{n}}$, Aric A. Prather, $\mathrm{PhD}^{\circ}$, Rebecca J. Schmidt, PhD ${ }^{\mathrm{p}}$, Karen A. Scott, MD, MPH, FACOG a,b,q, Solaire Spellen, MPH ${ }^{\mathrm{a}, \mathrm{b}}$, Jodi D Stookey, $\mathrm{PhD}^{\mathrm{r}}$, Martha Tesfalul, MD ${ }^{\mathrm{a}, \mathrm{b}}$, Larry Rand, MD ${ }^{\mathrm{a}, \mathrm{b}}$, Laura L. Jelliffe-Pawlowski, PhD, MS ${ }^{\text {b,m }}$

\footnotetext{
${ }^{a}$ Department of Obstetrics, Gynecology \& Reproductive Sciences, University of California, San Francisco 490 Illinois Street, Flr 9 Box 2930, San Francisco, CA 94143, USA

${ }^{\mathrm{b}}$ California Preterm Birth Initiative, University of California, San Francisco, 490 Illinois Street, Flr 9 Box 2930, San Francisco, CA 94143, USA

${ }^{c}$ Department of Pediatrics, University of California San Diego, 9500 Gilman Drive, La Jolla, California 92093, USA

d Department of Family Health Care Nursing, 2 Koret Way, San Francisco CA 94143, USA

e Department of Environmental Health Sciences, Columbia University Mailman School of Public Health, 722 West 168th St. NY, NY 10032, USA

${ }^{\mathrm{f}}$ Department of Biological and Medical Informatics, University of California San Francisco, 550 16th Street, Flr 3, San Francisco, CA, 94158, USA

${ }^{g}$ Osher Center for Integrative Medicine, University of California, San Francisco, 1545 Divisadero St, San Francisco, CA 94115, USA

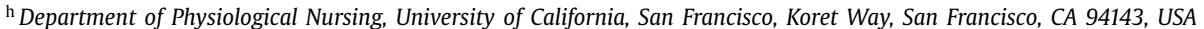

i Center for Learning \& Innovation, San Francisco Department of Public Health 25 Van Ness, Suite 500, San Francisco, CA, 94102, USA

jSchool of Social Welfare, Sexual Health and Reproductive Equity Program, University of California, Berkeley, 110 Haviland Hall MC 7400, Berkeley, CA 94720-7400, USA

${ }^{\mathrm{k}}$ Department of Pediatrics, University of California San Francisco, 550 16th Street, Box 0110, San Francisco, CA 94143, USA

${ }^{1}$ Department of Genetics, Stanford University School of Medicine, 291 Campus Drive, Stanford, CA 94305, USA

${ }^{m}$ Department of Epidemiology and Biostatistics, University of California, San Francisco, 550 16th Street, Flr 3, San Francisco, CA, 94158, USA

${ }^{\mathrm{n}}$ Newborn Brain Research Institute, Department of Pediatrics, Weill Institute for Neuroscience, University of California San Francisco, 16514 th St, San

Francisco, CA 94158, USA

${ }^{\circ}$ Department of Psychiatry and Behavioral Sciences, University of California, San Francisco, 3333 California Street, San Francisco CA 94118, USA

p Department of Public Health Sciences and MIND Institute, School of Medicine, and Perinatal Origins of Disparities Center; University of California Davis, 123 Med Sci 1C, One Shields Ave, Davis, California 95616, USA

${ }^{\mathrm{q}}$ Department of Humanities and Social Sciences, University of California, San Francisco, 490 Illinois Street, Flr 7, San Francisco, CA 94143, USA

${ }^{\mathrm{r}}$ Maternal, Child E Adolescent Health, San Francisco Department of Public Health, 30 Van Ness Avenue, Suite 260B, San Francisco, CA, USA
}

\section{A R T I C L E I N F O}

\section{Article history:}

Received 28 May 2021

Revised 12 July 2021

Accepted 12 July 2021

Available online 30 July 2021

Keywords:

COVID-19

Preterm Birth

Pregnancy

Racial/Ethnic Inequities

\begin{abstract}
A B S T R A C T
Introduction: Our understanding of the association between coronavirus disease 19 (COVID-19) and preterm or early term birth among racially and ethnically diverse populations and people with chronic medical conditions is limited.

Methods: We determined the association between COVID-19 and preterm (PTB) birth among live births documented by California Vital Statistics birth certificates between July 2020 and January 2021 $(n=240,147)$. We used best obstetric estimate of gestational age to classify births as very preterm (VPTB, $<32$ weeks), PTB (< 37 weeks), early term (37 and 38 weeks), and term (39-44 weeks), as each confer independent risks to infant health and development. Separately, we calculated the joint effects of COVID-19 diagnosis, hypertension, diabetes, and obesity on PTB and VPTB.
\end{abstract}

\footnotetext{
* Corresponding author:

E-mail address: deborah.karasek@ucsf.edu (D. Karasek).
} 
Findings: COVID-19 diagnoses on birth certificates increased for all racial/ethnic groups between July 2020 and January 2021 and were highest for American Indian/Alaska Native (12.9\%), Native Hawaiian/Pacific Islander (11.4\%), and Latinx (10.3\%) birthing people. COVID-19 diagnosis was associated with an increased risk of VPTB (aRR 1.6, 95\% CI [1.4, 1.9]), PTB (aRR 1.4, 95\% CI [1.3, 1.4]), and early term birth (aRR 1.1, 95\% $\mathrm{CI}[1.1,1.2])$. There was no effect modification of the overall association by race/ethnicity or insurance status. COVID-19 diagnosis was associated with elevated risk of PTB in people with hypertension, diabetes, and/or obesity.

Interpretation: In a large population-based study, COVID-19 diagnosis increased the risk of VPTB, PTB, and early term birth, particularly among people with medical comorbidities. Considering increased circulation of COVID-19 variants, preventative measures, including vaccination, should be prioritized for birthing persons.

Funding: UCSF-Kaiser Department of Research Building Interdisciplinary Research Careers in Women's Health Program (BIRCWH) National Institute of Child Health and Human Development (NICHD) and the Office of Research on Women's Health (ORWH) [K12 HD052163] and the California Preterm Birth Initiative, funded by Marc and Lynn Benioff.

(c) 2021 The Authors. Published by Elsevier Ltd. This is an open access article under the CC BY-NC-ND license (http://creativecommons.org/licenses/by-nc-nd/4.0/)

Research in context

\section{Evidence before this study}

Pregnant people are considered a high-risk population for COVID-19 infection, with evidence of the implication for birth outcomes emerging. Preterm birth (PTB, gestational age at birth $<37$ weeks) is one of the strongest determinants of child health and development and, therefore, an important outcome to consider in the context of COVID-19 diagnosis. While evidence from cohort studies and surveillance also suggest SARS-CoV-2 infection during pregnancy increases the risk of adverse birth outcomes, including PTB, less is known regarding how this relationship might vary by type of preterm birth. In a recent international multicenter cohort study, Villar et al found that COVID-19 diagnosis increased PTB by $59 \%$, driven primarily by medically-indicated rather than spontaneous PTBs. To date we lack evidence from a large US population-based cohort that allows examination by race/ethnicity and socioeconomic status. This is an important undertaking when we have seen dramatic inequities in the burden of COVID-19 infection and death.

\section{Added value of this study}

Using birth certificate data from all live births in California, we found that COVID-19 diagnoses increased for all racial/ethnic groups between July 2020 and January 2021, with the highest rates among American Indian/Alaska Native (12.9\%), Native Hawaiian/Pacific Islander (11.4\%), and Latinx $(10.3 \%)$ birthing people. COVID-19 diagnosis was associated with an increased risk of very PTB (aRR 1.6, 95\% CI [1.4, 1.9]), PTB (aRR 1.4, 95\% CI [1.3, 1.4]), and early term birth (aRR 1.1, 95\% CI [1.1, 1.2]). There was no effect modification of the overall association by race/ethnicity or insurance status. COVID-19 diagnosis was associated with elevated risk of PTB in people with comorbidities.

\section{Implications of all the available evidence}

In our study, we found increasing prevalence of COVID19 diagnosis over the course of the pandemic, with higher prevalence overall among Latinx, Native American/Alaskan Native, and Hawaiian/Pacific islander birthing people. COVID19 diagnosis in pregnancy was associated with a $40 \%$ increased risk of preterm, a $60 \%$ increased risk of very preterm, and a $10 \%$ increased risk of early term birth. Policy and clinical decision-makers should consider these findings to prioritize the health of pregnant people and their infants.

\section{Background}

Pregnant people are a high-risk group for severe coronavirus disease (COVID-19) caused by severe acute respiratory syndrome coronavirus 2 (SARS-CoV-2) infection [1,2,3]. While evidence from cohort studies and surveillance also suggest SARS-CoV-2 infection during pregnancy increases the risk of adverse birth outcomes, including preterm birth (PTB, gestational age at birth $<37$ weeks), less is known regarding how this relationship might vary by type of PTB [2,4-6]. For example, in a recent international multicenter cohort study, Villar et al found that COVID-19 diagnosis increased PTB by $59 \%$, driven primarily by medically indicated rather than spontaneous PTBs [6]. Moreover, reporting on risk of very preterm birth (VPTB, $<32$ weeks), PTB ( $<37$ weeks), and early term birth (37-38 weeks) has been limited by study size, and little is known about how the association between COVID-19 and PTB may vary by race/ethnicity. Population-based estimates of COVID-19 diagnoses in pregnancy by race, ethnicity and insurance status are also limited. Data from Washington State suggest a higher rate of infection among pregnant people compared to the general public, especially among Black, Indigenous and other people of color (BIPOC) [7].

Racial and ethnic inequities in COVID-19 in the general US population are well documented, with Black, Latinx, American Indian/Alaskan Native, and Pacific Islander populations bearing a disproportionate burden of COVID-19-related adverse outcomes, including mortality [8]. Inequities are also apparent by socioeconomic status (SES) [9], a marker of occupational and environmental exposure as well as access to resources. BIPOC and low SES populations experience the highest rates of adverse birth outcomes, including PTB [10], making the additional risk from COVID-19 of particular concern. The causes of both PTB and COVID-19 inequities are multifactorial and stem from racial capitalism and other structural and social inequities [11-14].

Research suggests an association between comorbidities, such as hypertension, diabetes, and obesity, and the severity of infection, and adverse birth outcomes [5]. Some researchers have argued that preexisting comorbidities may magnify the effect of SARS CoV-2 infection on adverse outcomes [6], an association that 
would be particularly important for BIPOC groups burdened by a higher prevalence of these conditions.

We use data from all live births in California between July 2020 and January 2021 to determine the association between COVID-19 diagnosis and PTB. Our objectives are to examine 1) the prevalence of COVID-19 diagnosis in pregnancy, 2) the association of COVID-19 diagnosis with VPTB, PTB, early term birth, and PTB subtypes, 3) differences in these outcomes by race/ethnicity and insurance status (as a proxy for SES), and 4) whether additional risk for adverse outcomes is conferred by comorbidities, including preexisting and gestational hypertension, diabetes, and obesity. Timely reporting on these patterns is essential to target COVID-19 treatment and prevention, as well as to inform decision-making regarding vaccination choices, clinical management, and resource allocation.

\section{Methods}

This retrospective cohort study includes all live births between July 2020 and January 2021 documented by California Vital Statistics birth certificates, using data received by the California Preterm Birth Initiative by March 1, 2021. Births from hospitals that did not report any birthing people with COVID-19 diagnosis during the study period were excluded ( $n=4,277$ births from 26 hospitals; Supplemental Figure 1 describes the sample selection steps). The study followed the Strengthening the Reporting of Observational studies in Epidemiology guidelines [16].

\subsection{COVID-19 diagnosis}

COVID-19 diagnosis among birthing persons was reported on birth certificates as 'confirmed' or 'presumptive' starting in July 2020 , largely based on universal laboratory screening at admission at hospitals for birth. "Confirmed" indicates the test was confirmed by the Centers for Disease Control and Prevention (CDC) laboratory, and "presumptive" indicates that a state or local laboratory conducted the testing without CDC laboratory confirmation [17]. Although International Classification of Diseases (ICD) codes were not used (instead an indication on the birth certificate), for the purposes of these analyses, both confirmed and presumptive cases are considered diagnosed with COVID-19, in alignment with ICD10 guidelines [17] No information about the timing of infection or severity of illness was available. Therefore, testing may have occurred at hospital admission or earlier in pregnancy.

\subsection{Outcomes}

We used best obstetric estimate of gestational age to classify births as very preterm ( $<32$ weeks), preterm ( $<37$ weeks), early term ( 37 and 38 weeks), and term (39-44 weeks). We stratified outcomes into early term birth, PTB, and VPTB, as each confers increasing risk of infant morbidity and development [18]. We categorized PTB as spontaneous, provider-initiated for a medical indication, or unknown. Spontaneous PTB included preterm premature rupture of the membranes (PPROM), premature spontaneous labor with intact membranes, or tocolytic medication documented on the birth certificate. Provider-initiated PTB included those without PPROM, premature labor, or tocolytic administration who had a code for "medical induction" or "artificial rupture of membranes," or "cesarean birth" on the birth certificate. PTB without any of the aforementioned codes did not have an indication and were classified as unknown. This classification system has been shown to accurately identify $80 \%$ of PTB [19].

\subsection{Statistical analysis}

The rate of COVID-19 diagnosis was calculated for each month overall, by payer at birth (Medicaid insurance compared to non- public insurance, including self-pay), and by race/ethnicity of the birthing parent (Latinx, Non-Latinx white, Black, Asian, American Indian/Alaska Native, Native Hawaiian/Pacific Islander, other race, two or more races, and not stated/unknown).

The Cochrane-Armitage test for trend was used to determine a significant change in rates over time. We modeled relative risks (RRs) using log-link binary regression with robust standard errors. Descriptive statistics were reported for cell sizes of 5 or greater for people with versus without SARS COV-2 infection. Subgroups with 5 or fewer observations were omitted for identification concerns. We conducted overall and race/ethnicity stratified analyses. As comorbidities may be on the pathway from race/ethnicity to PTB outcomes, we ran models adjusting for two covariate sets: 1) maternal age, maternal education, participation in the Special Supplemental Nutrition Program for Women, Infants, and Children (WIC), insured by Medicaid for prenatal care, adequacy of prenatal care, parity, maternal place of birth, pre-pregnancy body mass index, smoking status, previous cesarean birth, previous PTB, interpregnancy interval (IPI), and, 2) prior variables plus pre-pregnancy and gestational hypertension and diabetes. Finally, we also examined risk of PTB by subtype (spontaneous, indicated, unknown). Separately, we also calculated the joint effects of COVID-19 diagnosis, and comorbidities of hypertension, diabetes, and obesity (any) on VPTB, PTB and early term birth. We conducted a complete-case analysis.

\subsection{Sensitivity analysis}

We conduced sensitivity analyses restircted to laboratoryconfirmed COVID-19 diagnoses. We also clustered by birth hospital, to account for unmeasured institutional level factors.

We used Statistical Analysis Software version 9.4 (Cary, NC) for all analyses. The Committee for the Protection of Human Subjects (CPHS) within the Health and Human Services Agency of the State of California approved study protocols.

\subsection{Data statement}

Our approval from CPHS does not permit sharing of individual level data. Aggregate data when cell sizes are greater than five may be shared.

\subsection{Role of the funding source}

The funders of the study had no role in study design, data collection, data analysis, data interpretation, or writing of the report.

\section{Results}

The sample included 240,157 live births to pregnant people in California; 3.7\% reported a COVID-19 diagnosis in pregnancy $(n=8,957)$ and $96.3 \%$ did not report a COVID-19 diagnosis $(n=231,200) .47 .2 \%$ of the sample identified as Latinx and the remainder self-identified as non-Latinx white (26.8\%), Black (4.9\%), Asian (13.2\%), American Indian/Alaskan Native (.03\%), Hawaiian/Pacific Islander $(0.4 \%)$, or other, unknown, or two or more races $(7.3 \%)$. Three quarters (74.3\%) were between 18 - 34 years of age when their infant was born, and $40.3 \%$ had public insurance at that time. Compared to people without a COVID-19 diagnosis, those with COVID-19 were more likely to be Latinx (68.3\% with vs. 46.4\% without COVID-19), to have less than a high school education (19.4\% with vs. $10.1 \%$ without COVID-19), and to have been insured by Medicaid (56.7\% with vs. 39.7\% without COVID-19; Table 1 ).

The prevalence of COVID-19 diagnosis reported on birth certificates increased for all racial/ethnic groups between July 2020 and 
Table 1

Descriptive statistics for California live births July 2020 to January 2021.

\begin{tabular}{|c|c|c|c|c|c|c|}
\hline & \multicolumn{2}{|c|}{ Full sample } & \multicolumn{2}{|c|}{ No COVID-19 } & \multicolumn{2}{|c|}{ COVID-19 + } \\
\hline & $\mathrm{N} 240,157$ & $\%$ & $\mathbf{N} 231,200$ & \%96.3 & N8,957 & $\% 3.7$ \\
\hline \multicolumn{7}{|l|}{ Race/ethnicity } \\
\hline Latinx & 113,323 & 47.2 & 107,205 & 46.4 & 6,118 & 68.3 \\
\hline \multicolumn{7}{|l|}{ Non-Latinx } \\
\hline White & 64,402 & 26.8 & 63,059 & 27.3 & 1,343 & 15 \\
\hline Black & 11,823 & 4.9 & 11,447 & 5 & 376 & 4.2 \\
\hline Asian & 31,572 & 13.2 & 31,046 & 13.4 & 526 & 5.9 \\
\hline American Indian/Alaska Native & 727 & 0.3 & 699 & 0.3 & 28 & 0.3 \\
\hline Native Hawaiian/Pacific Islander & 944 & 0.4 & 896 & 0.4 & 48 & 0.5 \\
\hline Other & 239 & 0.1 & 229 & 0.1 & 10 & 0.1 \\
\hline Two or more races & 5,739 & 2.4 & 5,602 & 2.4 & 137 & 1.5 \\
\hline Not stated/Unknown & 11,388 & 4.8 & 11,017 & 4.8 & 371 & 4.1 \\
\hline \multicolumn{7}{|l|}{ Maternal age } \\
\hline$<18$ years & 2,041 & 0.9 & 1,901 & 0.8 & 140 & 1.6 \\
\hline $18-34$ years & 178,512 & 74.3 & 171,372 & 74.1 & 7,140 & 79.7 \\
\hline$>34$ years & 59,544 & 24.8 & 57,868 & 25 & 1,676 & 18.7 \\
\hline Missing & 60 & 0.0 & 59 & 0.0 & $*$ & \\
\hline \multicolumn{7}{|l|}{ Education } \\
\hline$<12$ years & 25,126 & 10.5 & 23,385 & 10.1 & 1,741 & 19.4 \\
\hline 12 years & 58,237 & 24.3 & 55,551 & 24 & 2,686 & 30 \\
\hline$>12$ years & 139,707 & 58.2 & 135,896 & 58.8 & 3,811 & 42.6 \\
\hline Missing & 17,087 & 7.1 & 16,368 & 7.1 & 719 & 8.0 \\
\hline \multicolumn{7}{|l|}{ Payer for delivery } \\
\hline Private & 127,016 & 52.9 & 123,724 & 53.5 & 3,292 & 36.8 \\
\hline Public & 96,794 & 40.3 & 91,715 & 39.7 & 5,079 & 56.7 \\
\hline Other/unknown & 16,347 & 6.8 & 15,761 & 6.8 & 586 & 6.5 \\
\hline Nulliparous & 94,884 & 39.5 & 91,810 & 39.7 & 3,074 & 34.3 \\
\hline \multicolumn{7}{|c|}{ Place of birth for person giving birth } \\
\hline US & 162,322 & 67.6 & 156,685 & 67.8 & 5,637 & 62.9 \\
\hline Mexico & 31,392 & 13.1 & 29,409 & 12.7 & 1,983 & 22.1 \\
\hline Other/unknown & 46,443 & 19.3 & 45,106 & 19.5 & 1,337 & 14.9 \\
\hline \multicolumn{7}{|l|}{ Adequacy of prenatal care } \\
\hline Adequate plus/adequate & 158,895 & 66.2 & 153,382 & 66.3 & 5,513 & 61.6 \\
\hline Intermediate & 49,432 & 20.6 & 47,493 & 20.5 & 1,939 & 21.7 \\
\hline Inadequate & 24,692 & 10.3 & 23,517 & 10.2 & 1,175 & 13.1 \\
\hline Missing & 7,138 & 3.0 & 6,808 & 2.9 & 330 & 3.7 \\
\hline \multicolumn{7}{|l|}{ Month of birth } \\
\hline \multicolumn{7}{|l|}{2020} \\
\hline July & 36,516 & 15.2 & 35,588 & 15.4 & 928 & 10.4 \\
\hline Aug & 36,452 & 15.2 & 35,453 & 15.3 & 999 & 11.2 \\
\hline Sept & 35,703 & 14.9 & 34,840 & 15.1 & 863 & 9.6 \\
\hline Oct & 35,277 & 14.7 & 34,526 & 14.9 & 751 & 8.4 \\
\hline Nov & 32,714 & 13.6 & 31,748 & 13.7 & 966 & 10.8 \\
\hline Dec & 32,430 & 13.5 & 30,273 & 13.1 & 2,157 & 24.1 \\
\hline \multicolumn{7}{|l|}{2021} \\
\hline Jan & 31,065 & 12.9 & 28,772 & 12.5 & 2,293 & 25.6 \\
\hline
\end{tabular}

* Cells not displayed when $\mathrm{n}<5$

January $2021(\mathrm{p}<.0006)$. In January 2021 proportions were highest for Latinx (10.3\%), American Indian/Alaska Native (12.9\%), and Native Hawaiian/Pacific Islander (11.4\%) birthing people, and lowest among non-Latinx white (4.6\%) and Asian (3.8\%) birthing people (Figure 1). The prevalence of COVID-19 diagnosis was higher among people with Medicaid compared to those with other forms of insurance (e.g., January 2021: 9.6\% versus 5.9\%). Existing comorbidities were common in the cohort; $15.9 \%$ of the sample had hypertension, diabetes, obesity, or a combination of these comorbidities.

The PTB rate among COVID-19 diagnosed birthing people was $11.8 \%$ compared with $8.7 \%$ among those without a recorded COVID19 diagnosis (aRR 1.4, 95\% confidence interval (CI) [1.3, 1.4]; Figure 2). COVID-19 also was associated with increased risk of VPTB (aRR 1.6, 95\% CI [1.4, 1.9]), and early term birth (aRR 1.1, 95\% CI [1.1, 1.2]). Rates of PTB were statistically significantly higher among individuals with COVID-19 diagnoses across all racial/ethnic groups except among those identifying as Black (aRR 1.0, 95\% CI $[0.7,1.4]$ ) or Native Hawaiian/Pacific Islander (aRR 1.3, 95\% CI $[0.5,3.2])$. The highest risk of PTB was observed for American In-
dian/Alaskan Native birthing people with COVID-19 compared to those without (28.6\% vs. $12.3 \%$; aRR $2.7[1.3,5.7])$ as well as for Asian birthing people (12.0\% vs $8.4 \%$; aRR $1.6[1.3,2.1])$. Numbers of births were too small to examine VPTB for individuals identified as American Indian/Alaskan Native, Native Hawaiian/Pacific Islander, or as two or more races. Asian birthing people had the highest risk of VPTB with COVID-19 diagnosis (aRR 3.4 [2.1, 5.6]). There was no evidence for effect modification of the overall association by race/ethnicity ( $p=0.90$ ). COVID-19 diagnosis was associated with an elevated risk of VPTB, PTB, and early term birth among birthing people with public and non-public insurance, with little difference between the adjusted estimates between insurance types. The association of infection with PTB appeared similar for spontaneous (aRR 1.5, 95\% CI [1.2, 1.7), provider-initiated (aRR 1.3, 95\% CI [1.2, 1.4]), and unknown subtype (aRR 1.4, 95\% CI [1.2, 1.6]) (Figure 3). Models were unchanged or slightly attenuated with the inclusion of comorbidities in the covariate set (Table S1, Model 3).

COVID-19 diagnosis was associated with elevated risk of PTB in people with and without comorbidities, but those with hypertension, diabetes or obesity and COVID-19 diagnosis were at elevated 


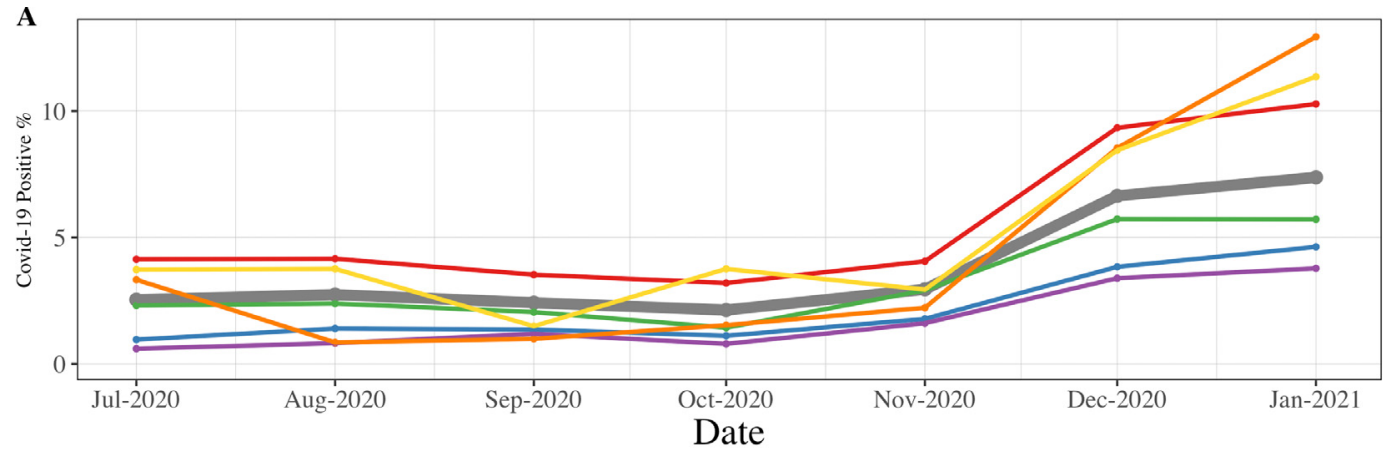

B

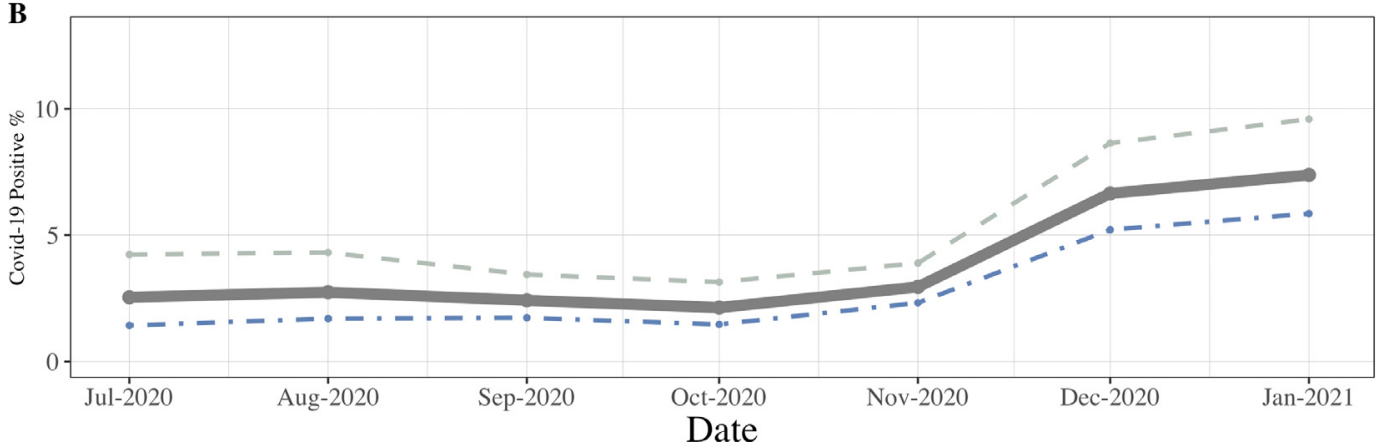

Race/Ethnicity

Overall

$\rightarrow$ Latinx

$\rightarrow$ White

$\rightarrow$ Black

- Asian

$\rightarrow$ American Indian/Alaska Native

$\sim$ Native Hawaiian/Pacific Islander

Insurance Status

Overall

$\rightarrow \quad$ Public Insurance

- Not Public Insurance

Figure 1. Prevalence of COVID-19 diagnosis for birthing people in California July 2020 to January 2021 by A) race/ethnicity and B) insurance.
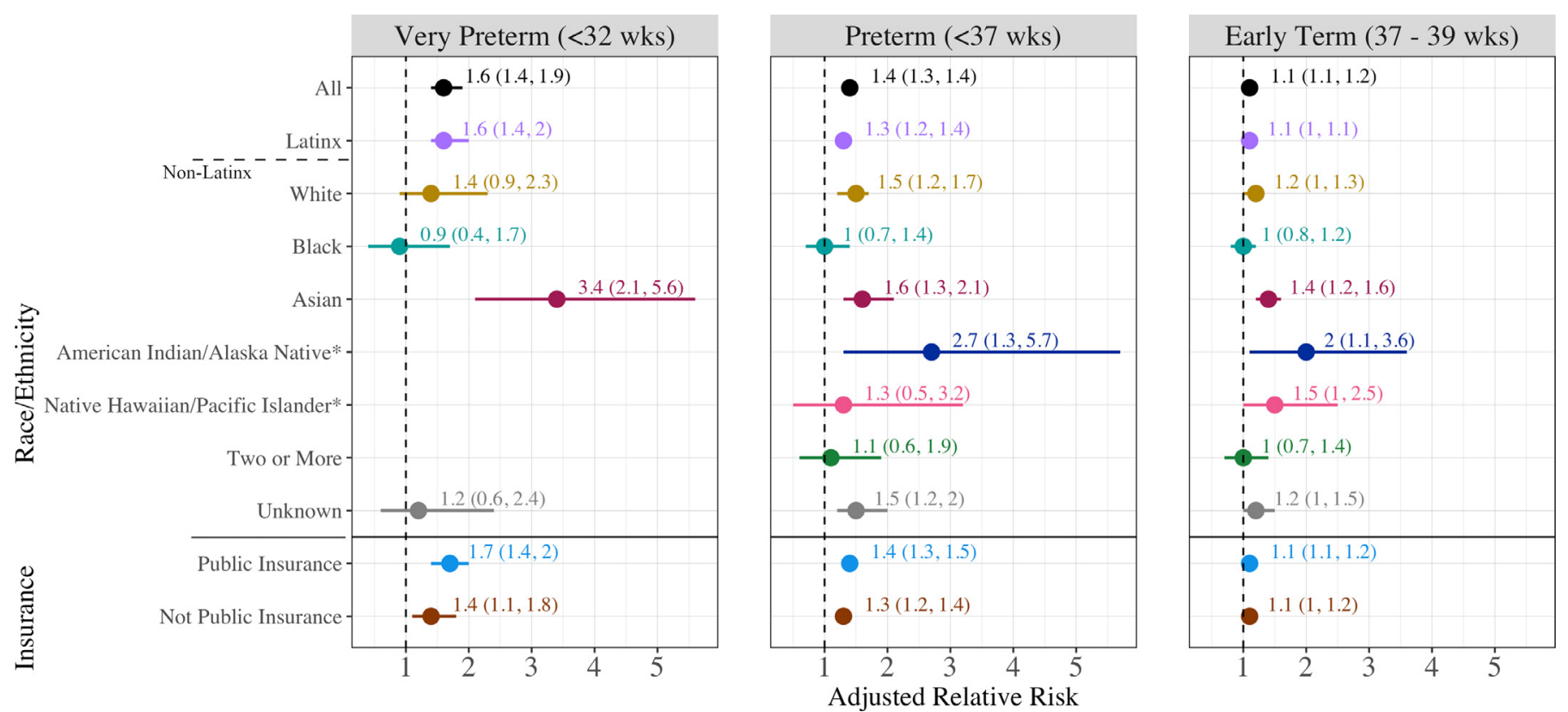

Figure 2. The association of COVID-19 diagnosis in pregnancy with very preterm, preterm and early term birth by race/ethnicity and insurance.

risk of VPTB (aRR 2.6, 95\% CI [2.1, 3.1]), PTB (aRR 2.0, 95\% CI [1.8, $2.2]$ ), and early term birth (aRR $1.3,95 \% \mathrm{CI}[1.2,1.4])$, compared to people without COVID-19 diagnosis or comorbidities (Table 2). The associations for individual comorbidities and COVID-19 diagnosis compared to those without a diagnosis or comorbidities are presented in the supplement (Table S2).

Sensitivity analyses restricted to COVID-19 cases with laboratory-confirmed results yielded similar results to our main models (Table S3). Additionally, adjusting for clustering at the birth hospital site did not alter the magnitude or precision of estimates.

\section{Discussion}

In this analysis, we examined the association between COVID19 diagnosis and PTB in a large population-based birth cohort in California $[8,20]$. To the best of our knowledge, this study represents the first study large enough to examine racial/ethnic and prematurity subgroups, demonstrating the increased risk of PTB in birthing people with COVID-19 diagnosis [4-6,21]. We provide critical new data on associations between COVID-19 diagnosis and VPTB, early term birth, PTB subtype, and by co-occurrence with other medical conditions, including hypertension, diabetes, and 

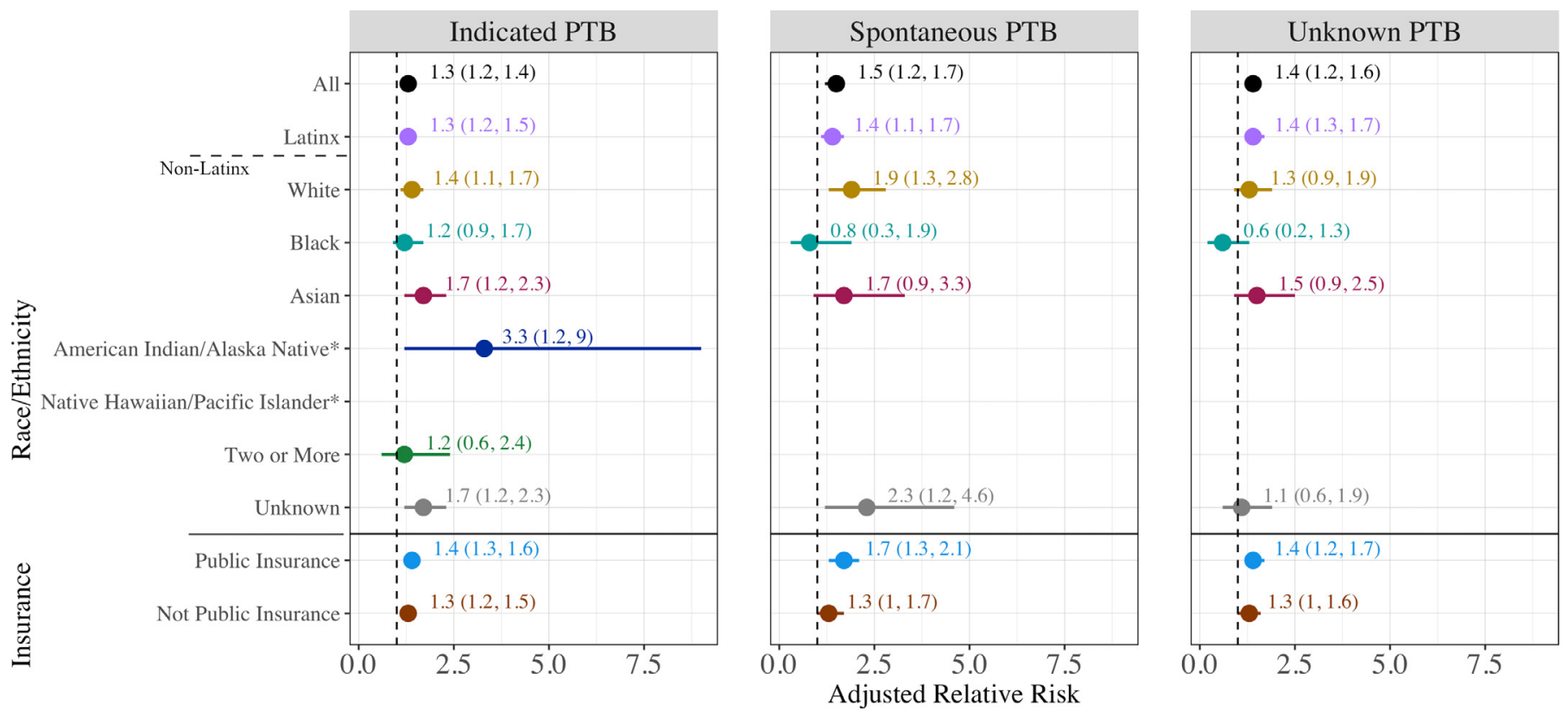

Figure 3. The association of COVID-19 diagnosis in pregnancy with preterm birth subtype - indicated, spontaneous or unknown.

Table 2

The association of COVID-19 diagnosis in pregnancy and comorbidities of hypertension, diabetes, or obesity with very preterm, preterm and early term birth. Models are: 1) unadjusted and 2) adjusted for maternal age, education, WIC participation, Medi-Cal for prenatal care, adequacy of prenatal care, parity, maternal place of birth, smoking, previous cesarean delivery, previous preterm birth, interpregnancy interval.

\begin{tabular}{|c|c|c|c|c|c|}
\hline & row $\mathbf{n}^{*}$ & $\begin{array}{l}\text { Very Preterm(< } 32 \text { weeks) } \\
\text { n (row \%) } \\
\text { Model } 1 \mathrm{RR}(95 \% \mathrm{CI}) \\
\text { Model } 2 \mathrm{aRR}(95 \% \mathrm{CI})\end{array}$ & $\begin{array}{l}\text { Preterm }(<\mathbf{3 7} \text { weeks) } \\
\text { n }(\text { row } \%) \\
\text { Model } 1 \mathrm{RR}(95 \% \mathrm{CI}) \\
\text { Model } 2 \text { aRR }(95 \% \mathrm{CI})\end{array}$ & $\begin{array}{l}\text { Early Term(37 - } 38 \text { weeks) } \\
\text { n (row \%) } \\
\text { Model } 1 \text { RR }(95 \% \mathrm{CI}) \\
\text { Model } 2 \text { aRR }(95 \% \mathrm{CI})\end{array}$ & $\begin{array}{l}\text { Term(39 - } 44 \text { weeks) } \\
\text { n (row \%) }\end{array}$ \\
\hline $\begin{array}{l}\text { All } \\
\text { No Hypertension, Diabetes or Obesity }\end{array}$ & 240,157 & $3,181(1.3)$ & $21,059(8.8)$ & $62,671(26.1)$ & $156,427(65.1)$ \\
\hline No COVID-19 & 146,114 & $1,556(1.1)$ & $10,362(7.1)$ & $34,873(23.9)$ & $\begin{array}{l}100,879(69.0) \\
\text { Reference }^{\mathrm{a}} \\
\text { Reference }^{\mathrm{a}}\end{array}$ \\
\hline COVID-19 + & 5,089 & $\begin{array}{l}87(1.7) \\
1.7(1.4,2.2) \\
1.6(1.3,2.0)\end{array}$ & $\begin{array}{l}488(9.6) \\
1.4(1.3,1.6) \\
1.3(1.2,1.5)\end{array}$ & $\begin{array}{l}1,400(27.5) \\
1.2(1.1,1.3) \\
1.1(1.1,1.2)\end{array}$ & $3,201(62.9)$ \\
\hline Hypertension, Diabetes or Obesity & & & & & \\
\hline No COVID-19 & 85,086 & $\begin{array}{l}1,442(1.7) \\
1.8(1.7,2.0) \\
1.7(1.6,1.9)\end{array}$ & $\begin{array}{l}9,637(11.3) \\
1.7(1.7,1.8) \\
1.6(1.6,1.7)\end{array}$ & $\begin{array}{l}25,225(29.7) \\
1.3(1.3,1.3) \\
1.3(1.2,1.3)\end{array}$ & $50,224(59.0)$ \\
\hline COVID-19 + & 3,868 & $\begin{array}{l}96(2.5) \\
2.8(2.3,3.5) \\
2.6(2.1,3.1)\end{array}$ & $\begin{array}{l}572(14.8) \\
2.3(2.1,2.5) \\
2.0(1.8,2.2)\end{array}$ & $\begin{array}{l}1,173(30.3) \\
1.4(1.3,1.5) \\
1.3(1.2,1.4)\end{array}$ & $2,123(54.9)$ \\
\hline
\end{tabular}

$* \mathrm{n}>100 \%$ because very preterm ( $<32$ weeks) births are also included in the preterm ( $<37$ weeks) births column

a For all

obesity. We found that COVID-19 diagnosis is associated with a $40 \%$ increased risk of PTB, a $60 \%$ increased risk of VPTB, and a $10 \%$ increased risk of early term birth. The heightened association for VPTB is especially concerning, as these births carry the highest risks of infant mortality and adverse outcomes [22]. Patterns of risk were similar across PTB subgroups, with a 50\% increased risk in spontaneous PTB, a $30 \%$ increased risk in provider-initiated PTB, and a $40 \%$ increased risk found for PTB with unknown subtype after adjustment for other known PTB risk factors. We did not see large differences in the relative risk in these models when we adjusted for comorbidities. The PTB subtype findings differ from previous studies that have only found an association in PTB classified as medically indicated [6]. The elevated risk for spontaneous delivery may indicate different mechanisms resulting in early birth.

Overall, we found evidence for a joint effect of hypertension, diabetes and/or obesity in pregnancy with COVID-19 infection in the production of very preterm and preterm birth. While the con- fidence intervals overlapped for most of the individual comorbidity and diagnosis estimates, they reveal the strong connection between pregnancy comorbidities preterm birth.

In addition, we found that, in California, COVID-19 diagnosis rates in pregnant people have continued to increase across all race/ethnicity groups but are disproportionately higher among Latinx, American Indian/Alaska Native, and Native Hawaiian/Pacific Islander birthing people, and among people with public insurance. Of note, while Latinx birthing people represented $47 \%$ of the overall sample, they represented $72 \%$ of the COVID-19 positive cases.

While we did not see evidence of a difference in the relative risk of COVID-19 diagnosis and PTB across all race/ethnicity groups, it is important to note that this investigation demonstrated the disproportionate burden of infection and PTB across Black, Indigenous, and other People of Color (BIPOC) groups. For example, while the rate of COVID-19 diagnosis among non-Latinx white birthing people in January 2021 was $4.6 \%$, it was $5.7 \%$ among Non-Latinx 
Black, $10.3 \%$ among Latinx, $11.4 \%$ among Native Hawaiian/Pacific Islander, and $12.9 \%$ among American Indian or Alaskan Native birthing people. Our results corroborate findings from a New York City study that found that pre-existing disparities in PTB and VPTB between Black and white infants were not further exacerbated during the pandemic [7]. The elevated risk of PTB with COVID-19 diagnosis for American Indian/Alaskan Native people highlights the disproportionate burden of infection and mortality experienced by this population $[23,24]$. Birthing people with public insurance appeared at similar risk of PTB from COVID-19 diagnosis than those with other insurance types.

These data point to the importance of continuing work to understand the impact of COVID-19 diagnosis on PTB and other outcomes, and to address higher prevalence of COVID-19 diagnosis in groups already facing inequities in birth outcomes as a result of structural racism. Of note, the patterns of COVID-19 diagnosis observed may have strong links to occupational exposures wherein racism and capitalism fundamentally shape COVID-19 disease inequity under the concept of racial capitalism [13,14]. Differences in infection may represent neighborhood- and building-level risk resulting from historical patterns of inequities, as demonstrated in other studies [25-27]. These data provide further evidence that BIPOC and low-income pregnant populations should be prioritized in efforts aimed at reducing risk of infection and the impact of the pandemic (e.g., through policy protections, prioritized vaccination, rent control, and economic supports). These analyses examined the associations of presumed and confirmed COVID-19 diagnosis with birth outcomes, rather than the population-level impacts of the pandemic, which may reveal different trends $[15,28]$. The cooccurrence of shelter-in-place policies, economic uncertainty, unemployment, evictions, food insecurity, and increased childcare burden likely increased the stress and risk environment for pregnant people and should be the focus of future work.

Limitations of the analysis include lack of information about the timing of COVID-19 diagnosis during pregnancy and detailed information about the severity of infection. Pregnant people had to test positive at delivery or have a prior result documented in their record. COVID-19 diagnoses that occurred earlier in pregnancy were thus less likely to be captured, especially for pregnant people who may have sought less prenatal care during the pandemic. As work in this area progresses, timing of exposure will be critical to elucidate associations with VPTB, PTB, and early term birth. Understanding severity is also essential, as it has been demonstrated to confer increased risk in previous studies [5,6]. Previous work found that three quarters of patients identified from universal screening had asymptotic infection [4]. Thus our results suggest that asymptomatic infection may be conferring increased risk of early birth in addition to previously reported symptomatic infections. Similarly, we were limited in our examination of underlying medical conditions that may impact expression of COVID-19 and preterm birth risk. Future studies using electronic health records could include more detailed medical conditions. The use of birth certificate data that collapses Asian identified people into one category obfuscates the opportunity to understand inter-group differences that would have implications for targeted interventions/specific mitigation strategies. The birth certificate data do not allow for assessment of miscarriage, fetal loss, or abortion, which may have been impacted by SARS COV-2 infection and, as such, will be important for further investigation. These births occurred before vaccination became widely available, however recent studies have demonstrated the safety and immunogenicity of vaccination in pregnant people [29-31] These results, along with our findings of increased risk of PTB with COVID-19 diagnosis allow providers and birthing people make informed decisions surrounding vaccination.

Our results establish that the prevalence of COVID-19 diagnoses during pregnancy rose in California as cases increased across the state through January of 2021 [32]. COVID-19 during pregnancy is associated with elevated risk of PTB overall, VPTB and early term birth. While we did not find evidence of a multiplicative effect, our results confirm critical racial/ethnic and socioeconomic inequities in both infection and PTB. The findings underscore the importance of adopting an equity strategy in addressing the systems and structures that exclude minoritized and marginalized groups from optimal access to and utilization of care to mitigate onset and/or severity of COVID-19 and adverse birth outcomes.

\section{Contributions}

All authors contributed to conceptualization, data interpretation and manuscript review and editing. LR was involved with conceptualization and funding acquisition. DK, RB, LJP, MM, MK, JF, KS, AB conducted literature searches. DK, RB, and LJP wrote original draft of the manuscript and were involved with formal analysis. $\mathrm{CM}$ and JAC gave input on specification of models and methodology. RB, SO, and LJP verified the underlying data and curated data. JC led visualization of results.

\section{Declaration of interests}

The authors have no conflicts of interest to disclose.

\section{Data Availability}

Our approval from Committee for the Protection of Human Subjects (CPHS) within the Health and Human Services Agency of the State of California does not permit sharing of individual level data. Aggregate data when cell sizes are greater than five may be shared.

\section{Supplementary materials}

Supplementary data associated with this article can be found, in the online version, at doi:10.1016/j.lana.2021.100027.

\section{References}

[1] Novel Coronavirus 2019 (COVID-19). https://www.acog.org/clinical/ clinical-guidance/practice-advisory/articles/2020/03/novel-coronavirus-2019 (accessed March 19, 2021).

[2] Zambrano LD, Ellington S, Strid P, et al. Update: Characteristics of Symptomatic Women of Reproductive Age with Laboratory-Confirmed SARS-CoV-2 Infection by Pregnancy Status - United States. MMWR Morb Mortal Wkly Rep January 22-October 3, 2020 2020;69:1641-7.

[3] Delahoy MJ, Whitaker M, O'Halloran A, et al. Characteristics and Maternal and Birth Outcomes of Hospitalized Pregnant Women with Laboratory-Confirmed COVID-19 - COVID-NET, 13 States. MMWR Morb Mortal Wkly Rep March 1-August 22, 2020 2020:69:1347-54.

[4] Allotey J, Stallings E, Bonet M, et al. Clinical manifestations, risk factors, and maternal and perinatal outcomes of coronavirus disease 2019 in pregnancy: living systematic review and meta-analysis. BMJ 2020;370:m3320.

[5] Metz TD, Clifton RG, Hughes BL, et al. Disease Severity and Perinatal Outcomes of Pregnant Patients With Coronavirus Disease 2019 (COVID-19). Obstetrics \& Gynecology 2021 Latest Articles: 10.1097/AOG.0000000000004339.

[6] Villar J, Ariff S, Gunier RB, et al. Maternal and Neonatal Morbidity and Mortality Among Pregnant Women With and Without COVID-19 Infection: The INTERCOVID Multinational Cohort Study. JAMA Pediatrics 2021 published online April 22. doi:10.1001/jamapediatrics.2021.1050.

[7] Lokken EM, Taylor GG, Huebner EM, et al. Higher severe acute respiratory syndrome coronavirus 2 infection rate in pregnant patients. American Journal of Obstetrics and Gynecology 2021:S0002937821000983.

[8] Webb Hooper M, Nápoles AM, Pérez-Stable EJ. COVID-19 and Racial/Ethnic Disparities. JAMA 2020;323:2466.

[9] Clouston SAP, Natale G, Link BG. Socioeconomic inequalities in the spread of coronavirus-19 in the United States: A examination of the emergence of social inequalities. Soc Sci Med 2021:268:113554.

[10] Martin JA, Hamilton BE, Osterman MJK. Births in the United States. NCHS Data Brief 2019:1-8 2020;

[11] Malawa Z, Gaarde J, Spellen S. Racism as a Root Cause Approach: A New Framework. Pediatrics 2021;147. doi:10.1542/peds.2020-015602. 
[12] Bailey ZD, Krieger N, Agénor M, Graves J, Linos N, Bassett MT. Structural racism and health inequities in the USA: evidence and interventions. The Lancet 2017:389:1453-63.

[13] McClure ES, Vasudevan P, Bailey Z, Patel S, Robinson WR. Racial Capitalism Within Public Health-How Occupational Settings Drive COVID-19 Disparities. American Journal of Epidemiology 2020;189:1244-53.

[14] Laster Pirtle WN. Racial Capitalism: A Fundamental Cause of Novel Coronavirus (COVID-19) Pandemic Inequities in the United States. Health Educ Behav 2020;47:504-8.

[15] Janevic T, Glazer KB, Vieira L, et al. Racial/Ethnic Disparities in Very Preterm Birth and Preterm Birth Before and During the COVID-19 Pandemic. JAMA Netw Open 2021;4:e211816.

[16] von Elm E, Altman DG, Egger M, et al. Strengthening the Reporting of Observational Studies in Epidemiology (STROBE) statement: guidelines for reporting observational studies. BMJ 2007;335:806-8.

[17] Control C for D Prevention. ICD-10-CM Official Coding and Reporting Guidelines; 2020. through September 30, 2020. 2020. April 1, 2020.

[18] Sengupta S, Carrion V, Shelton J, et al. Adverse Neonatal Outcomes Associated With Early-Term Birth. JAMA Pediatr 2013;167:1053.

[19] Klebanoff MA, Yossef-Salameh L, Latimer C, et al. Development and Validation of an Algorithm to Determine Spontaneous versus Provider-Initiated Preterm Birth in US Vital Records. Paediatr Perinat Epidemiol 2016;30:134-40.

[20] Emeruwa UN, Spiegelman J, Ona S, et al. Influence of Race and Ethnicity on Severe Acute Respiratory Syndrome Coronavirus 2 (SARS-CoV-2) Infection Rates and Clinical Outcomes in Pregnancy. Obstetrics \& Gynecology 2020;136:1040-3.

[21] Woodworth KR, Olsen EO, Neelam V, et al. Birth and Infant Outcomes Following Laboratory-Confirmed SARS-CoV-2 Infection in Pregnancy - SET-NET, 16 Jurisdictions. MMWR Morb Mortal Wkly Rep 2020;69:1635-40 March 29-October 14, 2020.

[22] Institute of Medicine (US) Committee on Understanding Premature Birth and Assuring Healthy Outcomes. Preterm Birth: Causes, Consequences, and Preven- tion. Washington (DC): National Academies Press (US), 2007 http://www.ncbi. nlm.nih.gov/books/NBK11362/ (accessed Sept 3, 2019).

[23] Burki T. COVID-19 among American Indians and Alaska Natives. Lancet Infect Dis $2021 ; 21: 325-6$.

[24] Arrazola J. COVID-19 Mortality Among American Indian and Alaska Native Persons - 14 States. MMWR Morb Mortal Wkly Rep 2020;69 January-June 2020. doi:10.15585/mmwr.mm6949a3.

[25] Emeruwa UN, Ona S, Shaman JL, et al. Associations Between Built Environment, Neighborhood Socioeconomic Status, and SARS-CoV-2 Infection Among Pregnant Women in New York City. JAMA 2020;324:390.

[26] Nardone AL, Casey JA, Rudolph KE, Karasek D, Mujahid M, Morello-Frosch R. Associations between historical redlining and birth outcomes from 2006 through 2015 in California. PLoS ONE 2020;15:e0237241.

[27] S S-J, B B, S C, et al. Neighborhood Evictions, Marital/Cohabiting Status, and Preterm Birth among African American Women. Ethn Dis 2021;31:197-204.

[28] Harvey EM, McNeer E, McDonald MF, et al. Association of Preterm Birth Rate With COVID-19 Statewide Stay-at-Home Orders in Tennessee. JAMA Pediatrics 2021 published online March 15. doi:10.1001/jamapediatrics.2020.6512.

[29] Shanes ED, Otero S, Mithal LB, Mupanomunda CA, Miller ES, Goldstein JA. Severe Acute Respiratory Syndrome Coronavirus 2 (SARS-CoV-2) Vaccination in Pregnancy: Measures of Immunity and Placental Histopathology. Obstetrics \& Gynecology 2021 Latest Articles: 10.1097/AOG.0000000000004457.

[30] Collier AY, McMahan K, Yu J, et al. Immunogenicity of COVID-19 mRNA Vaccines in Pregnant and Lactating Women. JAMA 2021 published online May 13. doi:10.1001/jama.2021.7563.

[31] Shimabukuro TT, Kim SY, Myers TR, et al. Preliminary Findings of mRNA Covid-19 Vaccine Safety in Pregnant Persons. N Engl J Med 2021:NEJMoa2104983.

[32] California State. Tracking COVID-19 in California of. State of California; 2021. https://covid19.ca.gov/state-dashboard/ (accessed May 13). 